\title{
Growth mechanism of stacked-cone and smooth-surface GaN nanowires
}

\author{
X. M. Cai, A. B. Djurišić, and M. H. Xie ${ }^{a)}$ \\ Department of Physics, The University of Hong Kong, Pokfulam Road, Hong Kong \\ C. S. Chiu and S. Gwo \\ Department of Physics and Institute of Microelectromechanical System, National Tsing-Hua University, \\ Hsinchu 300, Taiwan
}

(Received 11 July 2005; accepted 12 September 2005; published online 25 October 2005)

\begin{abstract}
Gallium nitride $(\mathrm{GaN})$ nanowires were grown on uncoated or Ni-coated Si substrates by evaporation of $\mathrm{Ga}$ in $\mathrm{NH}_{3}$ / Ar gas flow. A mixture of nanowires growing along [10 $\left.\overline{1} 0\right]$ and [0001] was obtained, where the former showed smooth surfaces and the latter showed the stacked-cone morphology. The yield of the two types of nanowires was found to depend on the $\mathrm{NH}_{3}$ flow. The reduction of $\mathrm{NH}_{3}$ flow led to fewer stacked-cone nanowires. The growth mechanisms of the two types of GaN nanowires are discussed. (C) 2005 American Institute of Physics. [DOI: 10.1063/1.2126118]
\end{abstract}

$\mathrm{GaN}$ is an important material for optoelectronic applications. GaN nanowires were first synthesized using carbon nanotubes as the templates. ${ }^{1}$ Since then, several works on the growth of GaN nanowires by the vapor-liquid-solid ${ }^{2}$ (VLS) or vapor-solid ${ }^{3}$ methods have been reported. Progress has been made in the synthesis of ordered GaN nanowires with controlled cross section, length and diameter. ${ }^{4}$ Applications of GaN nanowires for single nanowire laser ${ }^{5}$ and field effect transistors have also been demonstrated. ${ }^{6}$

So far, the majority of reported GaN nanowires are smooth with constant radius. However, growth of stacked-cone ${ }^{7,8}$ and zigzag ${ }^{9} \mathrm{GaN}$ nanowires have also been reported recently. While the formation of zigzag nanowires may be due to kinking, ${ }^{10}$ the formation mechanism of stacked-cone nanostructures remains unknown. The commonly invoked hypotheses explaining the change of radius, such as an oscillation of supersaturation of the system, ${ }^{11,12}$ cannot fully account for the observed stacked-cone morphology of GaN nanowires. In this study, we follow the morphology of $\mathrm{GaN}$ nanowires grown under different $\mathrm{NH}_{3}$ flow rates and establish the morphological details of the various nanowires. Based on the results, we suggest a mechanism by which the smooth and stacked-cone GaN nanowires are formed.

The growth experiments were carried out in a standard tube furnace at $950{ }^{\circ} \mathrm{C}$. Clean or Ni-coated $\mathrm{Si}(100)$ and $\mathrm{Si}(111)$ substrates were placed in a tungsten boat, where 100 mg Ga (99.999\%) source was also present but $3 \mathrm{~mm}$ away from the substrates in the upstream. The substrates had been cleaned by sonication in toluene, acetone, ethanol, and deionized water. For Ni coating, the nickel film with a thickness of $\sim 10 \mathrm{~nm}$ was prepared by electron beam evaporation onto $\mathrm{Si}$ substrates [both $n$-type $\mathrm{Si}(100)$ and $p$-type $\mathrm{Si}(111)$ ], and then the desired patterns were fabricated by optical lithography. The lithography-patterned Si substrates were immersed into a nitric acid solution to selectively remove the unmasked nickel. A visible change of sample color was observed and indicated the removal of unmasked nickel. The tungsten boat was inserted in a quartz tube, which was pumped to $2.0 \times 10^{-1}$ Torr before being heated in a tube furnace. The temperature increased gradually at a rate of

\footnotetext{
${ }^{a)}$ Electronic mail: mhxie@ @kusua.hku.hk
}

$35^{\circ} \mathrm{C} / \mathrm{min}$ until it reached $950{ }^{\circ} \mathrm{C}$. It was then maintained at $950{ }^{\circ} \mathrm{C}$ for a period of $30 \mathrm{~min}$ under the flow of $\mathrm{NH}_{3}$ $(99.999 \%)$ and $\operatorname{Ar}(99.999 \%)$ mixture. The pressure of the quartz tube was 3.5 Torr. After $30 \mathrm{~min}$, the flow of $\mathrm{NH}_{3}$ and Ar gases were shut off and the temperature was let down naturally to room temperature. In this process, the quartz tube was kept in vacuum.

The samples were characterized with a variety of techniques, including scanning electron microscopy (SEM) (Leo 1530) equipped with the energy dispersive X-ray spectra $(\mathrm{EDX})$, conventional and high-resolution transmission electron microscopy (TEM) (JEOL 2010F) and selected area electron diffraction (SAED).

Figures 1(a), 1(b), and 1(c) show typical morphologies of the obtained GaN nanowires on Ni-coated Si(100). Similar results were also obtained on $\mathrm{Si}(111)$ substrates. The flow rates of $\mathrm{NH}_{3}$ and $\mathrm{Ar}$ were 25 and $50 \mathrm{sccm}$. Both smooth and stacked-cone structures are present, which are similar to the ones obtained by Peng et $a .^{7}$ and Bae et al. ${ }^{8}$ They are typically several micrometers long. From Fig. 1(c), one may note

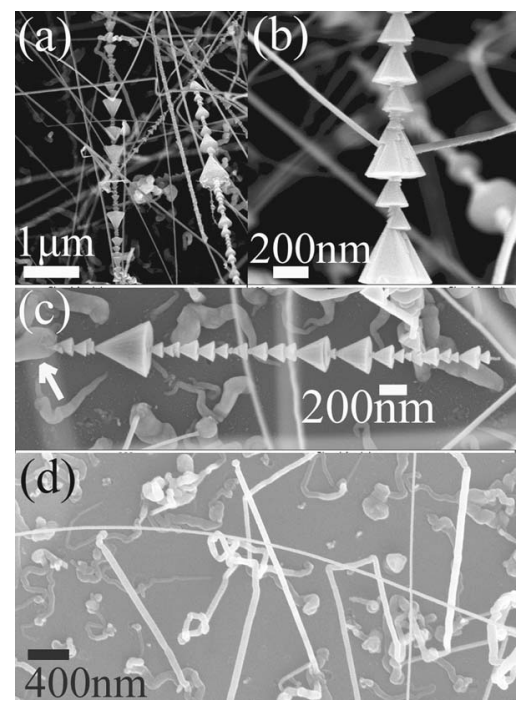

FIG. 1. (a) Typical morphology of the smooth surfaced and stacked-coneshaped GaN nanowires; (b) an enlarged image showing the stacked-cone GaN nanowire; (c) one stacked-cone GaN nanowire growing out from the catalytic droplet (pointed by the arrow); and (d) the typical morphology obtained by reducing $\mathrm{NH}_{3}$ while keeping the other parameters unchanged. 


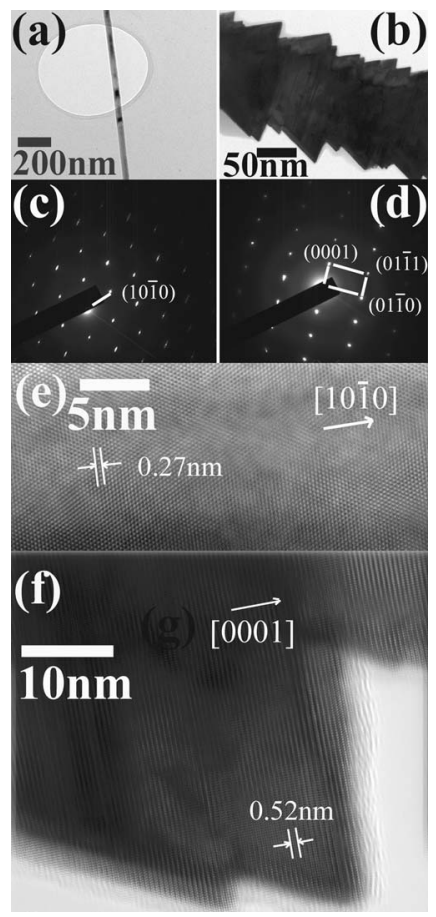

FIG. 2. (a), (c), and (e) represent the TEM image, diffraction pattern and high resolution image of the smooth surfaced nanowires; (b), (d), and (f) show the image, diffraction pattern and high-resolution image of the stacked-cone-shaped nanowire.

that the stacked-cone nanowires grow out from the catalytic droplet on the substrate and so it is quite plausible that it follows the tip VLS growth mode. ${ }^{13}$ EDX measurements of the stacked-cones show that they contain mainly Ga and N, suggesting that they are $\mathrm{GaN}$ nanowires. Measurements of the angles between the side faces and the bases in Fig. 1 give rise to values peaked at $\sim 62^{\circ}$, which implies that the side faces are of $\{10 \overline{1} 1\}$ planes. $^{7}$ The lateral sizes of the cones are measured to be about $400 \mathrm{~nm}$ for the bases while the tops are around $100 \mathrm{~nm}$. GaN nanowires with smooth surfaces, on the other hand, are likely grown via the base VLS mode, ${ }^{2}$ as indicated by the presence of particles at the ends of the nanowires. EDX measurements show that the particles at the nanowire ends contain $\mathrm{Ga}, \mathrm{N}$, as well as $\mathrm{Ni}$, whereas for the nanowire themselves, no $\mathrm{Ni}$ is detected.

Figure 1(d) shows the samples grown on Ni-coated $\mathrm{Si}$ substrate but with the gas flow rates of 10 and $65 \mathrm{sccm}$ for $\mathrm{NH}_{3}$ and $\mathrm{Ar}$, respectively. Note that the total flow rate of the gases and the pressure in the quartz tube were maintained the same as that used during growth of samples shown in Figs. 1(a)-1(c), but the flow rate of the $\mathrm{NH}_{3}$ gas is more than halved. It is observed from the SEM images of Fig. 1(d) that only GaN nanowires with smooth surfaces were obtained in this case. Reduction of $\mathrm{NH}_{3}$ to Ar flux ratio while the other growth parameters (including the total flow rate) remain unchanged simply leads to the reduced $\mathrm{N}$ supply. It thus seems to suggest that the more $\mathrm{N}$-rich condition favors the stackedcone morphology.

Figures 2(a) and 2(b) present the TEM micrographs of GaN nanowires with different morphologies, while Figs. 2(c) and 2(d) show the SAED patterns for the smooth-surfaced and stacked-cone nanostructures. The high-resolution TEM images of the two nanowires are given in Figs. 2(e) and 2(f). The diffraction pattern as well as the measured lattice spacDownloaded 19 Apr 2007 to 147.8.143.135. Redistribution subject

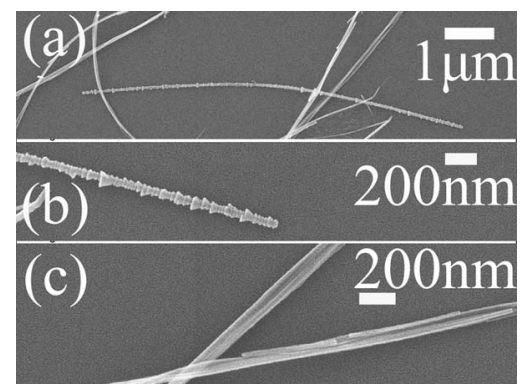

FIG. 3. (a) The typical morphology of the samples grown on $\mathrm{Si}$ without Ni catalyst; (b) high magnification of the stacked-cone nanowire in (a); and (c) high magnification of the nanobelt in (a).

ing along the wire growth direction confirm that the smoothsurfaced nanowires are GaN growing along $\langle 10 \overline{1} 0\rangle$, whereas the stacked-cones are growing along $\langle 0001\rangle$. The bases of the cones are $\{0001\}$ planes but the sidefaces are $\{10 \overline{1} 1\}$.

The stacked-cone morphology is also obtained on $\mathrm{Si}(100)$ and $\mathrm{Si}(111)$ substrates without Ni catalyst. Figure 3 shows an example grown under the gas flows of $125 \mathrm{sccm}$ for $\mathrm{NH}_{3}$ and $25 \mathrm{sccm}$ for Ar. Note that in addition to the stacked-cone morphology, GaN nanobelts were also observed. In all cases, however, we do not observe spherical droplets at the ends of nanowires and nanobelts when the catalyst is not used.

In the following, we offer an explanation for the formation of the observed morphologies of GaN nanowires. It has been known that the diffusivity of adatoms on different surfaces can be quite different and the presence of adsorbates will further change the characteristics of the surfaces. Recent total energy calculations as well as experimental results all confirmed the presence of surface excess $\mathrm{Ga}$ adlayers on GaN polar surfaces such as (0001) and (0001) so as to reduce the formation heat of the surfaces and also the energy barrier for adatom diffusion. ${ }^{14-17}$ Therefore, under Ga-rich growth condition, formation and expansion of such surfaces are preferred. This is achieved by growing $\mathrm{GaN}$ nanowires along $\langle 10 \overline{1} 0\rangle$ with $\{0001\}$ planes being the side faces of the nanowires. This explains the formation of nanobelts as firstly noted by Nam et al. ${ }^{3}$ For the smooth-surface nanowires with circular cross sections, they are likely limited by the spherical catalyst droplets. The smoothness of the sidefaces of the nanowires and nanobelts is consistent with the high diffusivity of adatoms on such surfaces covered by $\mathrm{Ga}$ adlayers, so there is an efficient mass transport of atoms from the sidefaces to the ends of the nanowires. Note that since the low formation energy of $\{0001\}$ faces and the high diffusivity of adatoms on such planes are directly related to the presence of excess Ga layers, nanobelts, and smooth-surfaced nanowires are more likely to form under Ga-rich conditions. This is consistent with our experimental observation that by reducing $\mathrm{NH}_{3}$ flow rates, more smooth-surface nanowires are obtained.

In less Ga-rich conditions, growth along $\langle 0001\rangle$ can be as likely as along $\langle 10 \overline{1} 0\rangle$ directions. ${ }^{3}$ Growth of nanowires along $\langle 0001\rangle$ leads to an expansion of the $\{10 \overline{1} 0\}$ planes as the sidefaces (Fig. 4). However, on such surfaces adatom diffusion is limited and so the surfaces easily become roughened, leading to GaN nanowires with coarse and corrugated sidesurfaces. ${ }^{3}$ Further roughening of the sidefaces leads to o AlP license or copyright, see http://apl.aip.org/apl/copyright.jsp 
(a) $\stackrel{\mathrm{Ga}}{\bullet} \mathrm{N}$

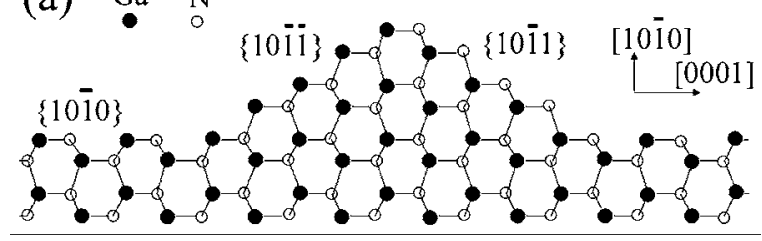

(b)

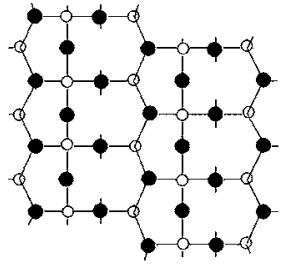

unreconstructed

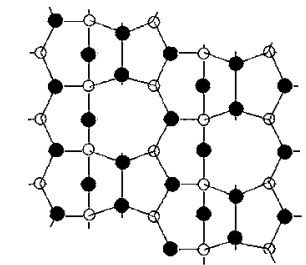

reconstructed

FIG. 4. Schematic diagram showing (a) Ga-polar $\{10 \overline{1} \overline{1}\}$ and N-polar $\{10 \overline{1} 1\}$ facets on GaN nanowire grown along [0001]; and (b) the Ga-polar $\{10 \overline{1} \overline{1}\}$ surface without (left panel) and with (right panel) surface reconstruction.

the development of $\{10 \overline{1} 1\}$ facets, which are polar and closepacked planes as illustrated in Fig. 4. Surface reconstruction by the dimerization of atoms readily occurs on these surfaces [see Fig. 4(b)], leading to further reduction of the energy of these surfaces. As has been reported during metalorganic vapor phase epitaxy of GaN under $\mathrm{N}$ rich conditions, $\{10 \overline{1} 1\}$ facets also appeared ${ }^{18}$ and so it seems that $\{10 \overline{1} 1\}$ faceting develops readily from surface roughening. To explain the cones rather than the more symmetrical structures, we note that $(10 \overline{1} 1)$ and $(10 \overline{1} \overline{1})$ planes have different polarities: one is Ga polar while the other is N-terminated (see Fig. 4). Not only that the two planes may show different formation energies but also that adatom diffusivity can be quite different. ${ }^{17}$ Therefore, one is more likely to develop at the expense of the other.

As for the apparent oscillation of the stacked cones, we believe they very likely develop from all the protrusions on the rough sidefaces of the wire. Due to the statistical nature of the roughening process, the protrusions will show different magnitudes and therefore, the sizes of the cones developed from them will also show variations. In an early experiment of Si whisker growth, the periodic oscillatory structure was also noted, which was attributed to the oscillation of supersaturation of the catalystic droplets ${ }^{11}$ Although we cannot rule out completely the relevance of such supersaturation oscillation to the stacked-cone formation, the facts that smooth wires as well as catalytic droplets connected by straight wire segments are also obtained in the same system point to other factors. Identification of these factors will require further study.

To conclude, we have observed GaN nanowires with both smooth sidefaces and the stacked-cone morphologies. Nanobelt or nanoribbon structures are also observed. The

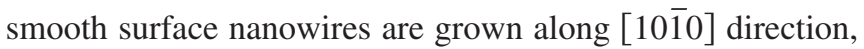
where $\{0001\}$ form part of the sidefaces of the nanostructures. For the stacked-cone structure, it is grown along [0001] and the sidefaces are $\{10 \overline{1} 1\}$ facets. The yield of different nanostructures depends on the flow rates of $\mathrm{NH}_{3}$ and Ar gases, where reducing the flow of $\mathrm{NH}_{3}$ leads to more smooth-surfaced nanowires. The formation of such nanostructures is understood in terms of the formation energies of different faces and the diffusivity of adatoms on different surface planes. The presence of excess Ga is important in the selection of nanowire morphologies.

The authors would like to thank Amy Wong and Wing Song Lee (Electron Microscopy Unit, HKU) for SEM measurements. This work is financially supported by grants from the Research Grant Council of the Hong Kong Special Administrative Region, China (Project Nos. HKU 7035/03P and HKU7019/04P) and the University of Hong Kong University Development Fund Grant.

${ }^{1}$ W. Q. Han, S. S. Fan, Q. Q. Li, and Y. D. Hu, Science 277, 1287 (1997).

${ }^{2}$ X. Duan and C. M. Lieber, J. Am. Chem. Soc. 122, 188 (2000).

${ }^{3}$ C. Y. Nam, D. Tham, and J. E. Fischer, Appl. Phys. Lett. 85, 5676 (2004).

${ }^{4}$ G. Kipshidze, B. Yavich, A. Chandolu, J. Yun, V. Kuryatkov, I. Ahmad,

D. Aurongzeb, M. Holtz, and H. Temkin, Appl. Phys. Lett. 86, 033104 (2005).

${ }^{5}$ J. C. Johnson, J. C. Heon, K. P. Knutsen, R. D. Schaller, P. D. Yang, and R. J. Saykally, Nat. Mater. 1, 106 (2002).

${ }^{6}$ Y. Huang, X. Duan, Y. Cui, and C. M. Lieber, Nano Lett. 2, 101 (2002).

${ }^{7}$ H. Y. Peng, N. Wang, X. T. Zhou, Y. F. Zheng, C. S. Lee, and S. T. Lee, Chem. Phys. Lett. 359, 241 (2002).

${ }^{8}$ S. Y. Bae, H. W. Seo, D. S. Han, M. S. Park, W. S. Jang, C. W. Na, J. Park, and C. S. Park, J. Cryst. Growth 258, 296 (2003).

${ }^{9}$ X. T. Zhou, T. K. Sham, Y. Y. Shan, X. F. Duan, S. T. Lee, and R. A. Rosenberg, J. Appl. Phys. 97, 104315 (2005).

${ }^{10}$ R. S. Wagner and C. J. Doherty, J. Electrochem. Soc. 115, 93 (1968).

${ }^{11}$ E. I. Givargizov, J. Cryst. Growth 20, 217 (1973).

${ }^{12}$ H. Kohno and S. Takeda, J. Cryst. Growth 216, 185 (2000).

${ }^{13}$ W. Q. Han and A. Zettl, Appl. Phys. Lett. 80, 303 (2002).

${ }^{14}$ A. R. Smith, R. M. Feenstra, D. W. Greve, J. Neugebauer, and J. E. Northrup, Phys. Rev. Lett. 79, 3934 (1997).

${ }^{15}$ S. H. Xu, Huasheng Wu, X. Q. Dai, W. P. Lau, L. X. Zheng, M. H. Xie, and S. Y. Tong, Phys. Rev. B 67, 125409 (2003).

${ }^{16}$ J. Neugebauer, T. K. Zywietz, M. Scheffler, J. E. Northrup, H. Chen, and R. M. Feenstra, Phys. Rev. Lett. 90, 056101 (2003).

${ }^{17}$ T. Zywietz, J. Neugebauer, and M. Scheffler, Appl. Phys. Lett. 73, 487 (1998).

${ }^{18}$ S. Kitamura, K. Hiramatsu, and N. Sawaki, Jpn. J. Appl. Phys., Part 2 34, L1184 (1995). 